\title{
Mapping climate disadvantage for care provision in London, UK: a sociospatial heat vulnerability assessment
}

\author{
E Oikonomou, R Raslan, A Dennett, J Taylor, P Symonds, A Mavrogianni
}

UCL Energy Institute (E Oikonomou MSc), UCL Institute for Environmental Design and Engineering (R Raslan PhD, J Taylor PhD, P Symonds PhD, A Mavrogianni PhD), and UCL Centre for Advance Spatial Analysis (A Dennett PhD), The Bartlett, University College London, London, UK

Correspondence to:

Ms Eleni Oikonomou, UCL Energy Institute, University College London, Central House, London WC1H ONN, UK

e.oikonomou@ucl.ac.uk,

\begin{abstract}
Background Climate change will increase the frequency and magnitude of extreme heat events, with major impacts for public health, communities, and the economy. The distribution of climaterelated disadvantage varies geographically as a function of public health policy, and of sociodemographic, building, and environmental factors. Elderly, socially isolated individuals and people with cardiovascular or respiratory diseases, depression, or low mobility are the most severely affected. The aim of this study was to accelerate development of equitable responses to climate change health risks for care provision in urban environments, with a focus on overheating risk.
\end{abstract}

Methods Using Greater London, UK, as a case study, we identified at-risk care homes by overlaying known locations of care homes onto areas of climate vulnerability identified by the Climate Just initiative. Built form and construction data were determined from building level energy rating schemes and other existing databases. These homes were cross-checked against a set of existing archetypes, developed as part of a previous research study, which are thought to be broadly representative of the London domestic stock. A meta-modelling framework was then built, based on the outputs of multiple EnergyPlus runs developed using artificial neural networks, specifically focusing on the prediction of indoor temperature conditions of common typologies of London care homes under warm weather conditions.

Findings Preliminary mapping of over $\mathbf{3 0 0}$ London care homes indicated that $\mathbf{6 3}$ were located in areas of extremely high or acute sociospatial vulnerability. Initial meta-modelling analysis results showed a significant variation in temperatures among indoor spaces located on different floor levels and/or facing different orientations, when the two-day mean maximum external temperature was between 28 and $30^{\circ} \mathrm{C}$. Top-floor rooms were the most prone to overheating and particularly those in buildings with a higher roof thermal efficiency. Overall, the higher the floor level and the roof insulation, the higher the mean daytime maximum temperature of the simulated domestic stock.

Interpretation The findings will have important implications for building designers, social care providers, and managers by helping map the potential costs associated with overheating risks in care settings and by outlining required changes to existing inspection processes.

This work will potentially inform public health policy guidance that aims to create care provision fit for the current and future climate. The findings and the methods used could be applied to other relevant population segments or geographical boundaries. 
Funding Bartlett Synergy grant 2017.

Contributors

AM led the project and the climate disadvantage mapping component. RR contributed to policy aspects of the project. AD led the spatial analysis work. JT and PS contributed the meta-modelling inputs. EO undertook meta-analysis of modelling data and mapping work. EO wrote the abstract with input from AM, RR, AD, JT and PS. All authors have seen and approved the final version of the abstract for publication.

Declaration of interests

We declare no competing interests. 